\title{
Design of an Enhanced Sensitivity FBG Strain Sensor and Application in Highway Bridge Engineering
}

\author{
Litong $\mathrm{LI}^{1,2^{*}}$, Dongsheng ZHANG ${ }^{1,2}$, Hui LIU ${ }^{1}$, Yongxing GUO ${ }^{1,2}$, and \\ Fangdong $\mathrm{ZHU}^{1,2}$ \\ ${ }^{1}$ National Engineering Laboratory for Fiber Optic Sensing Technology, Wuhan University of Technology, Wuhan, \\ 430070, China \\ ${ }^{2}$ Key Laboratory of Fiber Optic Sensing Technology and Information Processing (Ministry of Education), Wuhan \\ University of Technology, Wuhan, 430070, China \\ *Corresponding author: Litong LI E-mail: gnotilil@gmail.com
}

\begin{abstract}
The theoretical design method of enhanced sensitivity fiber grating (FBG) strain sensors was given, and moreover high qualified strain sensors were developed and fabricated, whose sensing properties were good for practical applications. The strain sensor with cylindrical shell encapsulation contained three tubular structures, due to the uneven surface structure, in the area of the strain concentration, improving the sensitivity. It could achieve the embedment strain measurement and surface measurement and had the advantages of the easy installation. The good agreement was obtained between the measurements and theoretical simulation results. After each calibration test, twenty-four FBG strain sensors and six FBG temperature compensation sensors have been installed on the undersurface of the box girder of Diaoshuiyan bridge in Yongtaiwen highway. Finally, we built up a long-term structure health system for the highway bridge.
\end{abstract}

Keywords: Fiber Bragg grating, strain sensors, bridge engineering

Citation: Litong LI, Dongsheng ZHANG, Hui LIU, Yongxing GUO, and Fangdong ZHU, "Design of an Enhanced Sensitivity FBG Strain Sensor and Application in Highway Bridge Engineering," Photonic Sensors, 2014, 4(2): $162-167$.

\section{Introduction}

The strain detection is an important physical quantity to evaluate the use and security situation of a highway bridge. In a traditional electric strain sensor, an electrical signal is the converted and transmitted carrier. Therefore, under the severe environment, it often brings out some problems. For example, the electromagnetic radiation can cause misinformation and inefficiency, too much humidity can cause a short circuit, and the high-temperature atmosphere can lead to fire. Meanwhile, serial electric sensors can not be connected with each other when the number of sensors is in a certain size. You need to put a lot of signal transmission cables and also need the appropriate size of the signal receiving and processing facilities, leading to an increase in the cost. In recent years, the fiber grating technology, which has been widely applied in various fields, has the following virtues: anti-electromagnetic radiation and convenient cascade of the signal without the aid of electricity on the spot $[1,2]$. However, the bare fiber Bragg grating can only stand 2000 to 3000 microstrain, which can not satisfy the need of practical monitoring of 
infrastructures, especially for the damage detection. On the other side, the sensing coefficient of the FBG is limited by the interrogator, and nowadays so far it can only detect 1 or 2 micro strain, which is not enough for the high-precision monitoring situation $[3,4]$. So it is very necessary to bring forward a practical design method to enhance the FBG strain sensor's sensitivity. Zhi Zhou [5] came up with an idea that an FBG sensor with the spring inside is able to enhance the range to $10000 \mu \varepsilon$. Ren Liang [6] proposed an FBG strain sensor which was clamped both the ends and miniature architectural models used in the seismic testing and dam model testing.

In this paper, aiming at improving the sensitivity of the FBG strain sensors with the uneven surface structure, the principle of high sensitivity of the sensor is analyzed in detail, and the test method of the FBG strain sensor is discussed. Furthermore, the design of the FBG strain sensors was applied in Ningbo, a long-term structural health monitoring system of Diaoshuiyan bridge in Yongtaiwen highway.

\section{Description of the sensor}

The configuration of the sensor is shown in Fig.1. The structure of the sensor consists three parts: a measuring tube and two fixed tubes. All of them were made of stainless steel. A threaded connection between the measuring tube and the fixed tube, a stretched FBG was fixed to the two ends of the cavity in the measuring tube by using the adhesive.

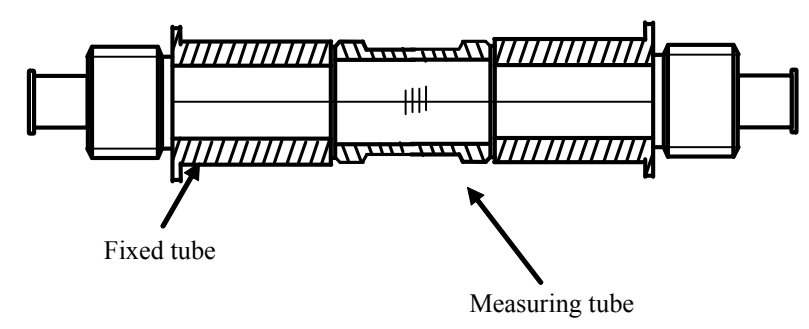

Fig. 1 Schematic structure of the FBG strain sensor.

The lengths of the measuring tube and the fixed tube are $l_{1}$ and $l_{2}$, while $s_{1}$ and $s_{2}$ are the cross-sectional areas of the measuring tube and the fixed tube. Also the test length of the sensor is $l$. Assume that $\Delta l_{1}, \Delta l_{2}$, and $\Delta l$ are the variations of the lengths $l_{1}, l_{2}$, and $l$. When the strain sensor is subjected to a tensile force $f$, the following equation is given:

$$
\Delta l=\Delta l_{1}+\Delta l_{2} .
$$

Assume that $\varepsilon_{1}, \varepsilon_{2}$, and $\varepsilon$ are the strain values distributed in the measuring tube, the fixed tube, and the test object. While $\varepsilon_{\mathrm{FBG}}$ is the test value of the FBG, which is equal to $\varepsilon_{1}$, the following equation is given

$$
\varepsilon l=\varepsilon_{1} l_{1}+\varepsilon_{2} l_{2} .
$$

The force distributed in the sensor is the same. $E$ is the Young's modulus of stainless steel, so the following equation is given:

$$
f=\varepsilon_{1} s_{1} E=\varepsilon_{\mathrm{FBG}} S_{1} E=\varepsilon_{2} s_{2} E .
$$

According to (2) and (3), the strain of the test object can be expressed as

$$
\varepsilon=\left(\frac{l_{1}}{l}+\frac{s_{1}}{s_{2}}-\frac{l_{1} s_{1}}{l s_{2}}\right) \varepsilon_{\mathrm{FBG}} .
$$

Equation (4) can be given by

$$
\varepsilon=\left(k_{1}+k_{2}-k_{1} k_{2}\right) \varepsilon_{\mathrm{FBG}}
$$

where $k_{1}=\frac{l_{1}}{l}$, and $k_{2}=\frac{s_{1}}{s_{2}}$. In the actual design of the sensor, $k_{1}=0.153$, and $k_{2}=0.352$. So $\frac{\varepsilon_{\mathrm{FBG}}}{\varepsilon}=2.34$.

The change in the Bragg wavelength is given by

$$
\Delta \lambda_{B}=\left(1-P_{e}\right) \lambda_{B} \varepsilon=K_{\varepsilon} \varepsilon
$$

where $P_{e}$ is the photo-elastic coefficient of the fiber, and $\varepsilon$ is the strain. For the silica single-mode fiber, $P_{e}=0.22$. The wavelength-strain sensitivity of the FBG in the silica fiber near $1550 \mathrm{~nm}$ is about $1.17 \mathrm{pm} / \mu \varepsilon$. In summary, the theoretical strain sensor strain sensitivity is $2.73 \mathrm{pm} / \mu \varepsilon$.

Therefore, the enhancing sensitivity of the sensor can be obtained by the above method. The 
strain distribution of the sensor under the force was modeled by the ANSYS finite element software. We set parameters of each strain gauge according to the material properties. Constraints and loads were imposed on the fixed parts of the two ends of the carrier. The strain distributions were simulated, as shown in Fig. 2. The concentrated strain was mainly on the measuring tube. Software simulation and numerical simulation matched very well.

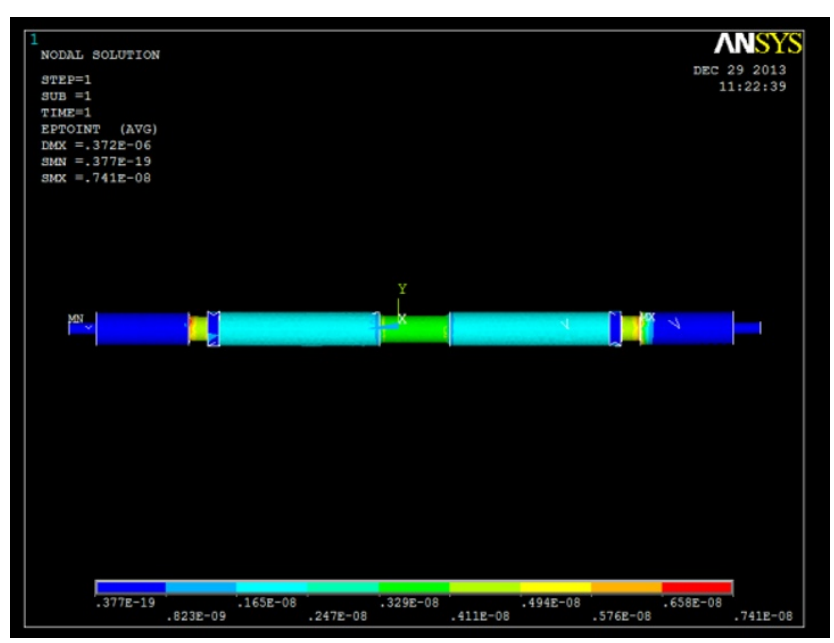

Fig. 2 Force analysis of the strain sensor.

\section{Experimental results and analysis}

Figure 3 is a photograph of the strain sensor. As shown in Fig. 4, in order to avoid the looseness of the connection between the measuring tube and the fixed tub, we used laser welding to fix it.

As shown in Fig. 5, we used a German "Zwick / Roell Z600E" universal testing machine to perform the test on the strain sensor. The testing machine was equipped with an automatic raster digital extensometer "Longstroke" (with the resolution of $1 \mu \mathrm{m})$.

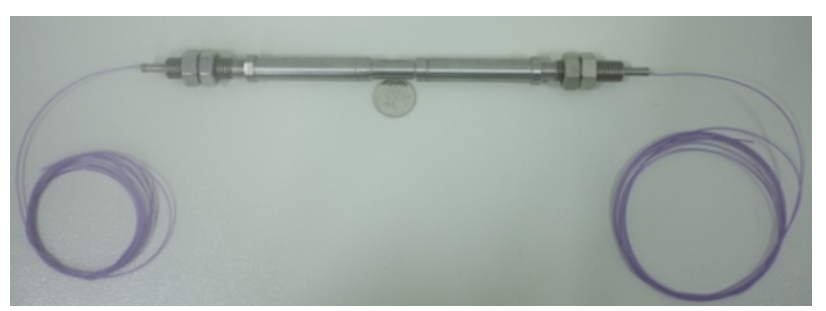

Fig. 3 Photo of the FBG strain sensor.

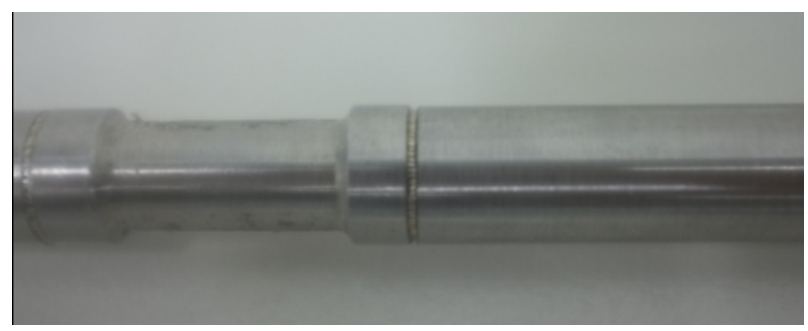

Fig. 4 Photo of the laser welding place in the sensor.

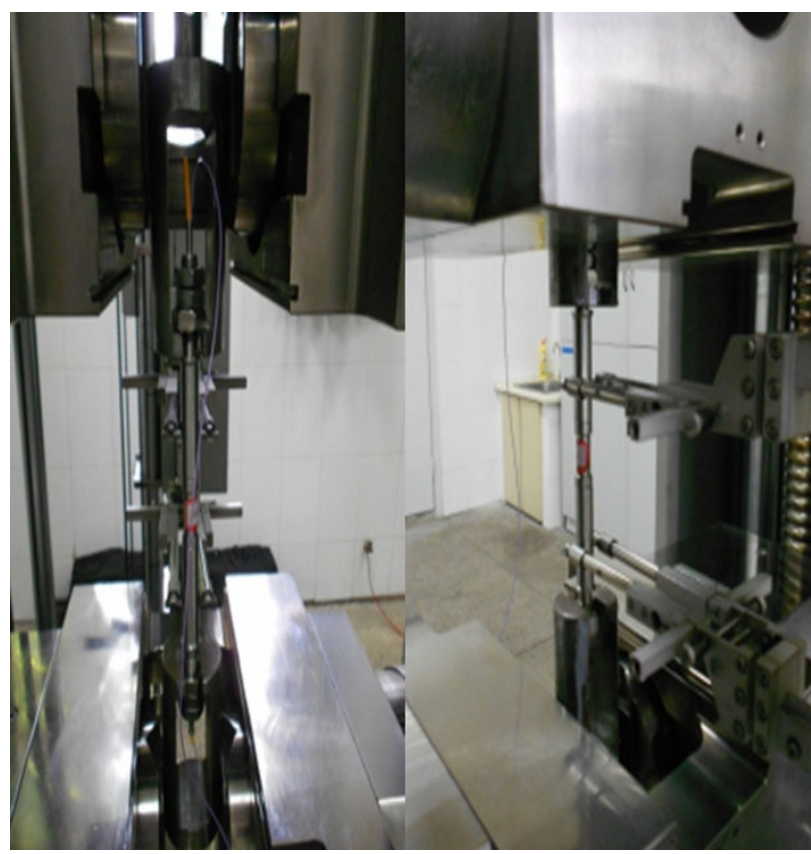

Fig. 5 Photograph of the experiment.

The tensile loading changed from $1 \mathrm{kN}$ to $4 \mathrm{kN}$ in steps of $0.5 \mathrm{kN}$, remaining at each step for at least 5 seconds and then slowly decreased to $0 \mathrm{kN}$. This process was repeated three times. Figure 6 is the length change results of the strain sensor during three tests. Figure 7 is the linear fit of the average length change. Figure 8 is the wavelength change results of the strain sensor during three tests. Figure 9 is the linear fit of the average wavelength change. From the figures below, after the calculation, we got that the sensitivity of the strain sensor was $2.52 \mathrm{pm} / \mu \varepsilon$. And the strain measurement test results were consistent with the theoretical calculation. For the long term monitoring system, we have produced 24 strain sensors. After the calibration test, the sensitivity of the sensors ranged from $2.4 \mathrm{pm} / \mu \varepsilon$ to $2.8 \mathrm{pm} / \mu \varepsilon$, both of which were consistent with the theoretical results. 


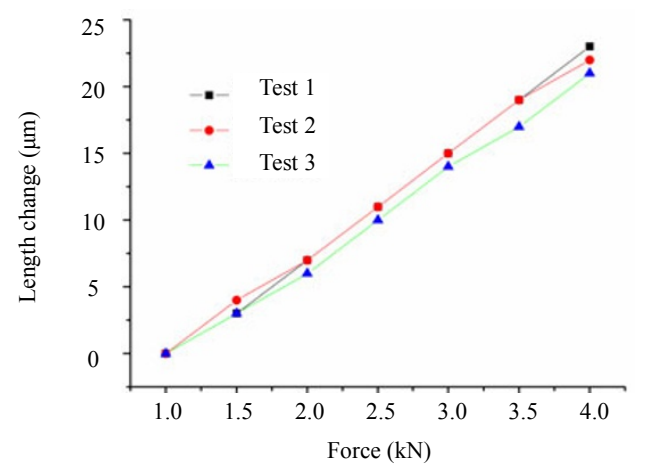
tests.

Fig. 6 Length change results of the strain sensor during three

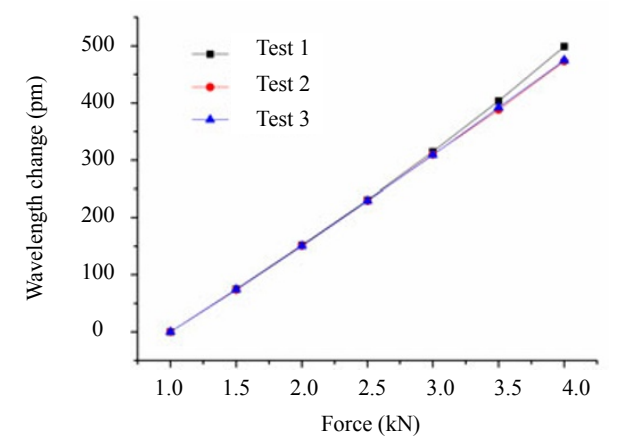

Fig. 7 Linear fit of average length change.

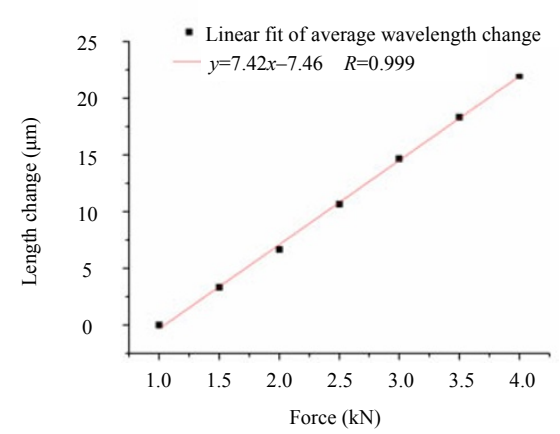

Fig. 8 Wavelength change results of the strain sensor during three tests.

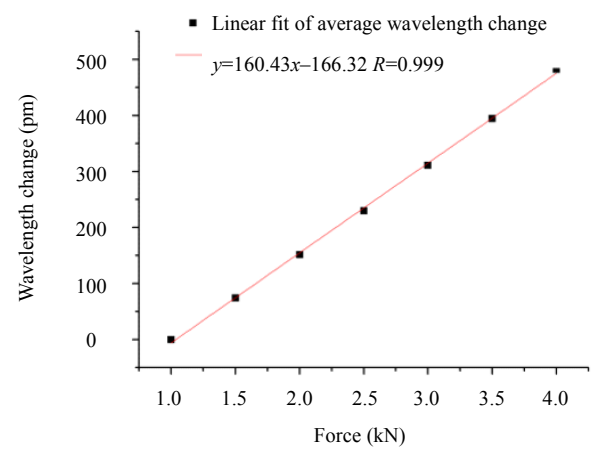

Fig. 9 Linear fit of the average length change.

\section{Long-term monitoring system}

Diaoshuiyan bridge in Yongtaiwen highway is located in Ningbo. The bridge structure is built in precast prestressed concrete box girder, across a total of four simple box girders, with each span length of 30 meters. After each calibration test, twenty-four FBG strain sensors and six FBG temperature compensation sensors have been installed on the undersurface of the first box girder of Diaoshuiyan bridge in Yongtaiwen highway.

The sensor installation diagram is shown in Fig. 10. Twenty four strain sensors were divided into three columns, respectively, arranged in the first span bridge bottom 1/4, 1/2, 3/4 points across, each column for a group of eight strain sensors. At the ends of each group, a temperature compensation sensor was installed [7, 8]. The wavelength of the sensors in each column was different, connected with each other. Then, the connected signals of all the sensors were sent into the interrogator device.

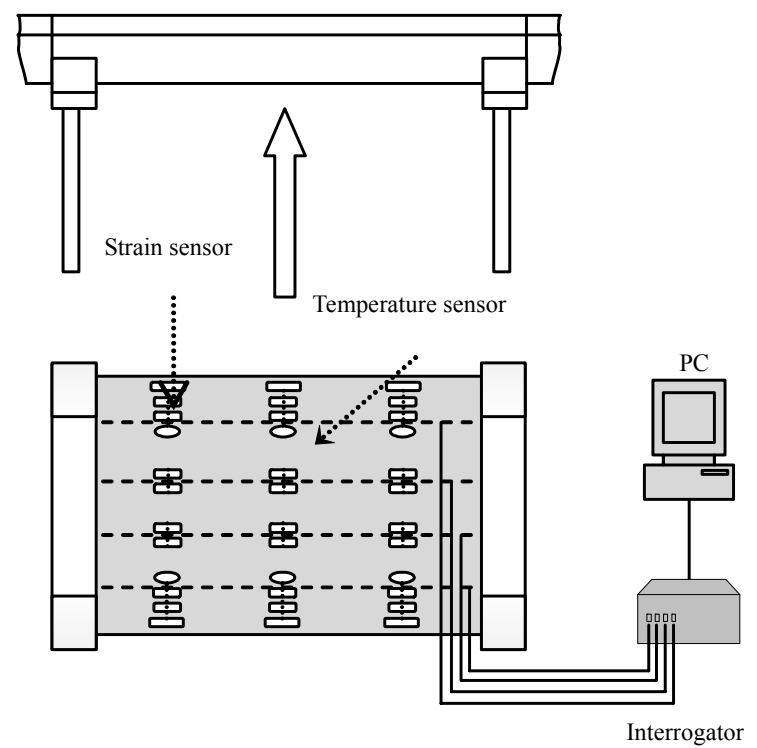

Fig. 10 Installation instruction of FBG strain sensors and temperature sensors.

As shown in Fig. 11, we used the set screw nut and fixed the damper to get the strain sensor fixed on the measured object. Figure 12 is the realistic diagram of sensors' installation. As shown in Fig. 13, the curve shows the measured strain values from one of the strain sensors during five months. Through experiments, it is proven that the sensing 
system is stable enough to meet drawing requirements.

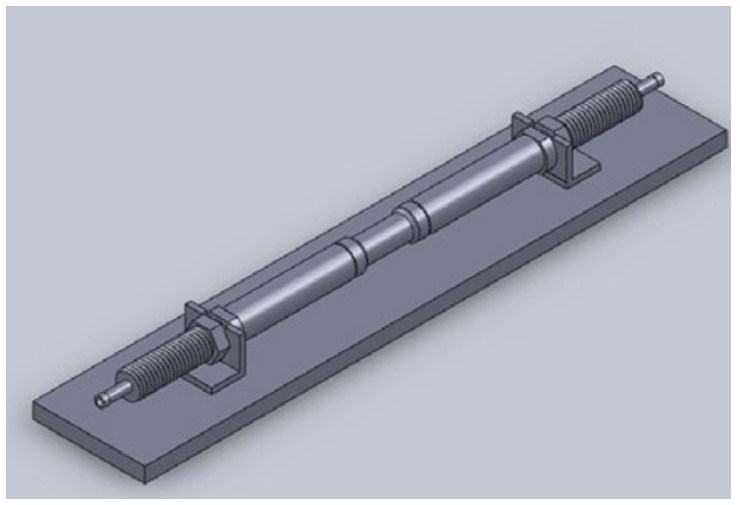

Fig. 11 Installation method of the strain sensor.

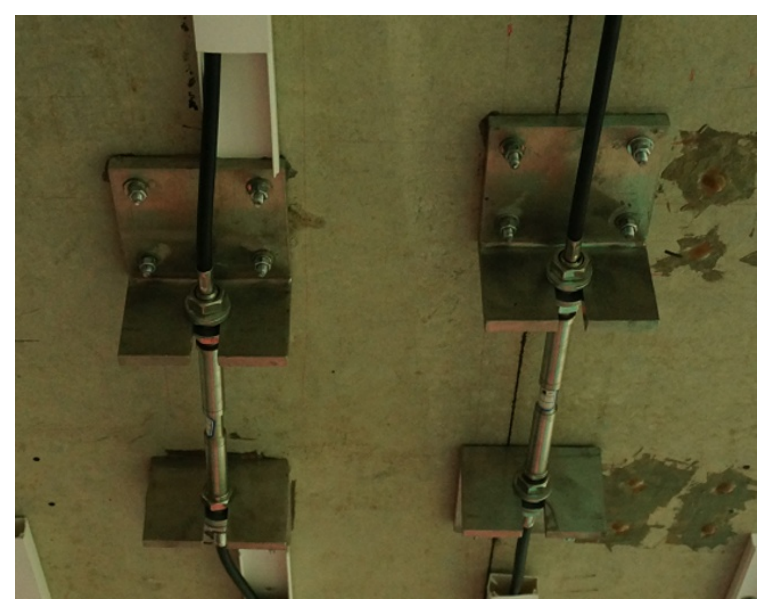

Fig. 12 Realistic diagram of sensors' installation.

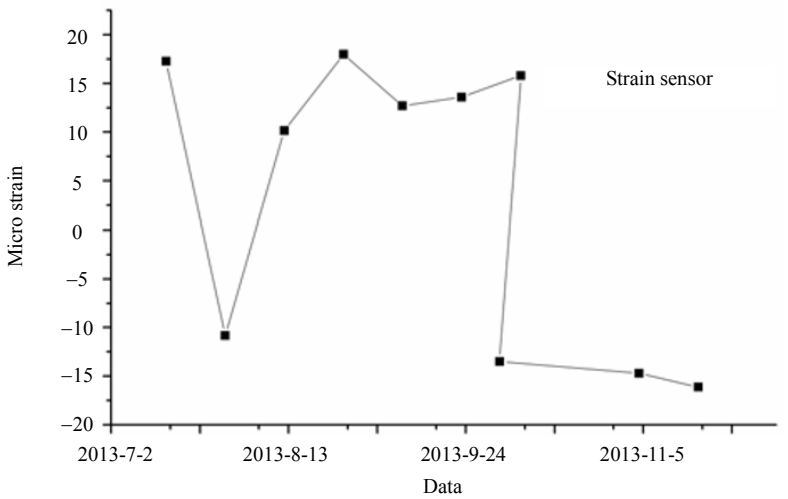

Fig. 13 Measured strain values from one of the strain sensors during five months.

\section{Conclusions}

In this research, we presented the structural design, performance test, and application of an enhanced sensitivity FBG strain sensor for the long-term structure health system of a highway bridge. Performance test results were consistent with the theoretical expectations of the sensor in the laboratory. After the test, twenty-four enhanced sensitivity FBG strain sensors and six FBG temperature sensors have been installed on the undersurface of the box girder of Diaoshuiyan bridge in Yongtaiwen highway. It can clearly and correctly detect the dynamic strain responses of the bridge during five months. This shows that the FBG sensor technology is a good alternative for civil and structural dynamic strain monitoring.

\section{Acknowledgment}

This work is supported in part by the National High Technology Research and Development Program (863 Program) of China (No: 2012AA041203), the Cultivation Fund of the Key Scientific and Technical Innovative Project, Ministry of Education of China (No: 708064).

Open Access This article is distributed under the terms of the Creative Commons Attribution License which permits any use, distribution, and reproduction in any medium, provided the original author(s) and source are credited.

\section{References}

[1] Y. J. Rao, "In-fibre Bragg grating sensors," Measurement Science and Technology, 1997, 8(4): 355-375.

[2] W. W. Morey, G. Meltz, and W. H. Glenn, "Fiber optic Bragg grating sensors", in Proc. SPIE, vol. 1169, pp. 98-107, 1989.

[3] W. Chung, S. Kim, and N. Kim, "Deflection estimation of a full scale prestressed concrete girder using long-gauge fiber optic sensors," Construction and Building Materials, 2008, 22(3): 394-401.

[4] H. Ling, K. Lau, and L. Cheng. "Determination of dynamic strain profile and delamination detection of composite structures using embedded multiplexed fibre-optic sensors," Composite Structures, 2004, 66(1-4): 317-326.

[5] Z. Zhou, J. He, J. He, and J. Ou, "Design method for coefficient-adjustable FBG strain sensors," Pacific 
Science Review, 2006, 8: 41-48.

[6] L. Ren, T. Jiang, and D. Li, "Application of small FBG strain sensors in dam model experiment," Journal of Vibration, Measurement \& Diagnosis, 2013, 33(2): 277-183.

[7] Y. Liu, Z Guo., Y. Zhang, K. S. Chiang, and X. Dong, "Simultaneous pressure and temperature measurement with polymer coated fiber Bragg grating," Electronics Letters, 2000, 36(6): 564-566.

[8] R. H. Scott, S. Chikermane, S. Srinivasan, P. A. M. Basheer, F. Surre, T. Sun, et al., "Commissioning and evaluation of a fiber-optic sensor system for bridge monitoring," IEEE Sensors Journal, 2013, 13(7): 2555-2562. 